\title{
OPG Fine Preoperative Evaluation of Maxillary Inflammatory and Atrophic Lesions with Postoperative Successfully Surgical Solutions Appreciation of Dentoalveollary Reconstructions Using Heterolog Membranes and Materials Toghether with Metallic Implants
}

\author{
BOGDAN ADRIAN SINK*, SILVIU BRAD², ALIN DORU TANASE ${ }^{3}$, ALEXANDRU BOGDAN BRAD4 \\ 'ESTETIQDENT, Dental Medicine Practice, 1301 Decembrie Str, 332008, Petrosani, Romania \\ 2 University of Medicine and Pharmacy Victor Babes, Department of General and Oral Radiology, 2 Eftimie Murgu Sq., \\ 300041, Timisoara, Romania \\ ${ }^{3}$ City Hospital, University of Medicine and Pharmacy Victor Babes, 2 Eftimie Murgu Sq., 300041, Timisoara, Romania \\ ${ }^{4}$ University of Medicine and Pharmacy Victor Babes, XV Department, 2 Eftimie Murgu Sq., 300041, Timisoara, Romania
}

\begin{abstract}
The purpose of this paper is to point out the value of OPG exam in pre and postoperative diagnosis assessment of maxillary inflammatory and atrophic lesions with the very full appreciation of surgical solutions and local integration of heterolog membranes and materials together with metallic implants used to repair the osseous defects and rehabilitate the dental status. There were statistically retrospective evaluated the pre and postoperative results of OPG exams of 47 patients with maxillary inflammatory and atrophic lesions clinically and biologically assessed, treated and dental rehabilitated by metallic implants at ESTETIQDENT, a dental medicine practice from Petrosani. In all cases we proceeded to surgical extraction of the non-vital teeth and ultrasonic plaques remove. In the presence of inflammatory radicular cysts, surgical radicular cysts removement and curettage of the periradicular granuloma were the elected choice together with heterolog membranes and materials repair of the osseous defects. When it was necessary, the existent prosthesis works were removed and in order to obtain the bone support, internal or external sinus lift and regional bone addition were performed for dental status rehabilitation through metallic implants. The OPG preoperative exams clearly showed the extent, the morphological characteristics and the topoanatomic reports, in all cases of maxillary inflammatory and atrophic lesions together with regional endodontic status. The postoperative OPG revealed the very local results of surgery procedures and the integration of heterolog membranes and materials used to repair the osseous defects and the dental status rehabilitation by metallic implants. The OPG exam is the imaging method of choice in pre and postoperative diagnosis assessment of maxillary inflammatory and atrophic lesions with the full appreciation of surgical solutions and local integration of heterolog membranes and materials together with metallic implants used to repair the osseous defects and rehabilitate the dental status, due to highly specific abilities in bone tissue evaluation of all maxillary skeletal and dental segments.
\end{abstract}

Keywords: inflammatory radicular cysts, maxillary atrophic lesions, heterolog membranes and materials, sinus lift, OPG exam

The dento-maxillary pathology is a major problem of medicine throughout the world due to the importance of this most anterior portion of the aero-digestive tract with essential functions in: speech, mastication, taste, deglutition, aesthetics and general well being. The pathological conditions within the teeth and jaw bones will affect the integrity of the oral cavity with serious implications of all this functions. Any local disease could be the cause of regional and general complications which concern the whole organism with a great impact on health population [1].

The inflammatory radicular cysts are by far the most common pathological conditions within the teeth and jaws bones that will affect the integrity of the oral cavity with serious implications of all his functions. The so common, inflammatory radicular cysts could be the cause of regional and general complications which concern the whole organism with a great impact on health population. Thus surgical radicular cysts removement and the associated non-vital tooth extraction are the treatment procedures of choice, with heterolog membranes and materials repairement of the osseous defect and dental rehabilitation by metallic implants as the required next steps $[1,2]$.
The alveolar crest atrophy after dental segments extraction is one of the most frequent condition of maxillary skeletal loss, reducing the height of alveolar ridge and thus hindering the installation of the implants required to the prosthetic support. In order to obtain the necessary bone support, there are many surgical reconstruction solutions to increase bone volume of atrophic jaws by maxillary sinus lifting or regional alveolar crest augmentation by bone addition.

The biocompatible materials used to repair the maxillary skeletal defects are natural - autogenic (autologous bone), allogenic (bone substitute from bone bank: demineralised freeze-dried bone allograft and demineralised bone matrix) or xenogenic (bone graft from bovine, equine, porcine or the exoskeleton of coral which are chemically deproteinised but with intact mineral structure) and totally synthetic or alloplastic also divided into: polymers, ceramics, metallic and composites [6].

Examination and diagnosis of inflammatory radicular cysts and alveolar atrophic crest by radiological and imaging modalities is the immediate of choice algorithm after clinical evaluation. Not only with the teeth and their

*email: sink_bogdan@yahoo.com, Phone: 0727/099807 
surrounding bone the oral radiology concern, but also with the entire oral and maxillofacial complex [3, 4].

Among the imaging techniques of dental radiology the most common are OPG and periapical radiographies together with the classical projections used in the diagnosis of maxilla and mandible pathology $[4,5]$. Due to the ability of high-quality multiplanar reconstructions the Multislice CT and CBCT offer performance in detailed oral cavity imaging.

The biocompatible materials used to repair the maxillary skeletal defects are natural - autogenic (autologous bone), allogenic (bone substitute from bone bank: demineralised freeze-dried bone allograft and demineralised bone matrix) or xenogenic (bone graft from bovine, equine, porcine or the exoskeleton of coral which are chemically deproteinised but with intact mineral structure) and totally synthetic or alloplastic also divided into: polymers, ceramics, metallic and composites [6].

Due to the ability of assessment of both the bone maxillary structures and the regional endodontic status OPG exam has proved to be an excellent procedure for characterizing the skeletal anatomy and related abnormalities of all oral regions being the current imaging modality of choice in pre and posttherapeutic daily dental medicine practice $[2,3]$.

The aim of this paper is to point out the value of OPG exam in pre and postoperative diagnosis assessment of inflammatory radicular cysts and alveolar atrophic crest together with regional oral cavity extent, bone repair and dental rehabilitation by metallic implants evaluation.

\section{Experimental part}

\section{Materials and methods}

We retrospectively reviewed the pre and postoperative results of OPG exams and periapical $x$-ray examinations of 47 patients with maxillary inflammatory and atrophic lesions clinically and biologically assessed, treated and dental rehabilitated by metallic implants at ESTETIQDENT, a dental medicine practice from Petrosani.

In all cases we proceeded to surgical extraction of the non-vital teeth and ultrasonic plaques remove. In the
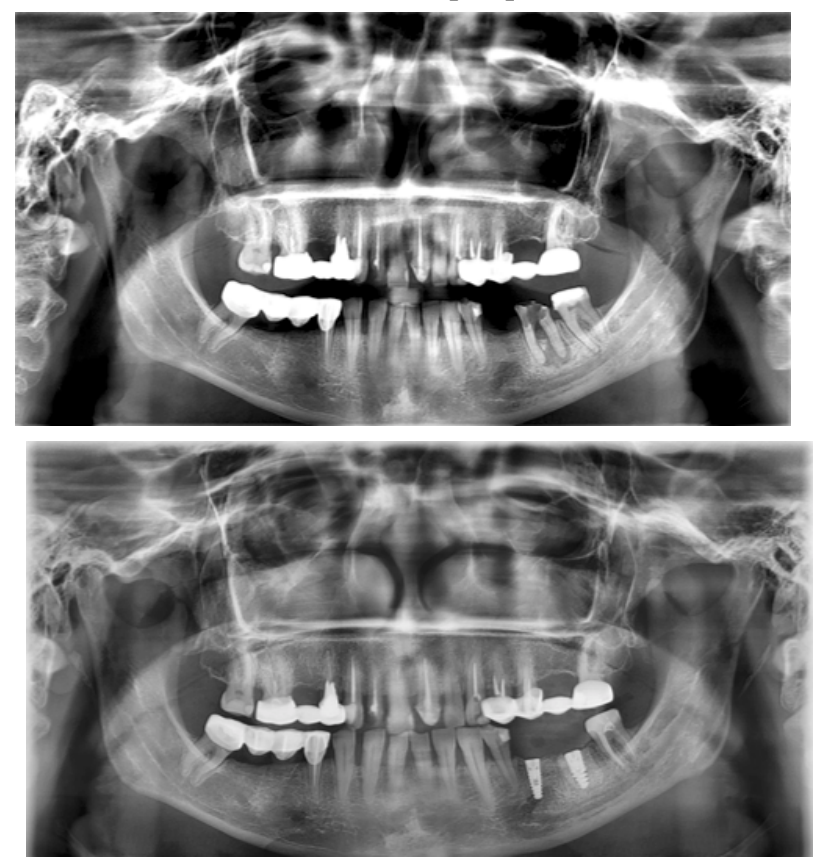

Fig.1. OPG exams in a pre and postoperative case of left posterior mandible 3.6 inflammatory radicular cysts with surgical removal, bovine bone addition repairement of the osseous defect and dental status rehabilitation through metallic implants presence of inflammatory radicular cysts, surgical radicular cysts remove and curettage of the periradicular granuloma were the elected choice together with heterolog membranes and materials repair of the osseous defects. When it was necessary, the existent prosthesis works were removed and in order to obtain the bone support, internal or external sinus lift and regional bone addition were performed for dental status rehabilitation through metallic implants. Surgical reconstruction of bone loss were done heterolog membranes and materials: bovine bone addition - Cerabone - granulation 1-2 mm which were then covered with a resorbable membrane of collagen - Osgide resorbable porcine membrane over which the gum suture is performed.

All patients were pre and postoperative X-ray examined by periapical dental radiographies obtained after the classical projection techniques protocol and by panoramic radiographies achieved with the usual orthopantomographic procedure.

Preoperative and postterapeutic surgical and grafting skeletal and dental procedures OPG exams were done with the same unit in order to precise regional oral cavity extent and dento-maxillary rehabilitation evaluation.

\section{Results and discussions}

From the 47 patients dental rehabilitated by metallic implants 18 patients were diagnostic assessed with inflammatory radicular cysts from which, 12 cases $(67 \%)$ were detected in the maxilla and 6 cases have mandible localization (33\%). In maxillary regions 1 case $(8 \%)$ was revealed in aesthetic anterior regions and in 11 cases (92\%) the sites were in non aesthetic posterior regions: 4 in the right (34\%) and 7 in the left (58\%). In mandible all the 6 cases (33\%) were found to have non aesthetic posterior localization: 4 in the right (67\%) and 2 in the left (33\%). Upon patients sex distribution the 12 maxillary radicular cysts were revealed in 7 cases in women (58\%) and in only 5 cases in men (42\%) and in 6 mandible localisations 4 cases were detected in men (67\%) and only 2 cases in women $(33 \%)$.

In all cases radicular cysts remove and the associated non-vital teeth extractions were the treatment procedures of choice with the repair of the osseous defect by bovine bone addition - Cerabone - granulation 1-2 mm which is then covered with a resorbable membrane of collagen Osgide - resorbable porcine membrane over which the gum suture is performed.

In all patients periapical radiographies and OPG exams revealed complete bone lysis done by inflammatory radicular cysts, guide the surgical therapy and finally appreciate the heterolog membranes and materials repair of the postoperative osseous defect.

All 47 patients dental rehabilitated by metallic implants were diagnostic assessed with regional alveolar crest atrophy after dental segments extraction.

In order to obtain the maxillary bone support for dental implant insert, external maxillary sinus lifting was necessary in 21 patients (60\%) and internal maxillary sinus lifting were done in 14 patients (40\%).

From 21 external maxillary sinuses lifting, 5 were done in both I and II quadrants (35\%), 4 in quadrant I (21\%) and 7 in quadrant II (44\%). Upon patient sex distribution, 12 external maxillary sinus lifting were done in women patients (57\%) and only 9 in men (43\%).

From 14 internal maxillary sinuses lifting 3 were done in both I and II quadrants (27\%), 5 in quadrant I (46\%) and 3 in quadrant II (27\%). Internal sinus lifting was done with Osteo Safe Anthogyr France (the first automatic osteotome 

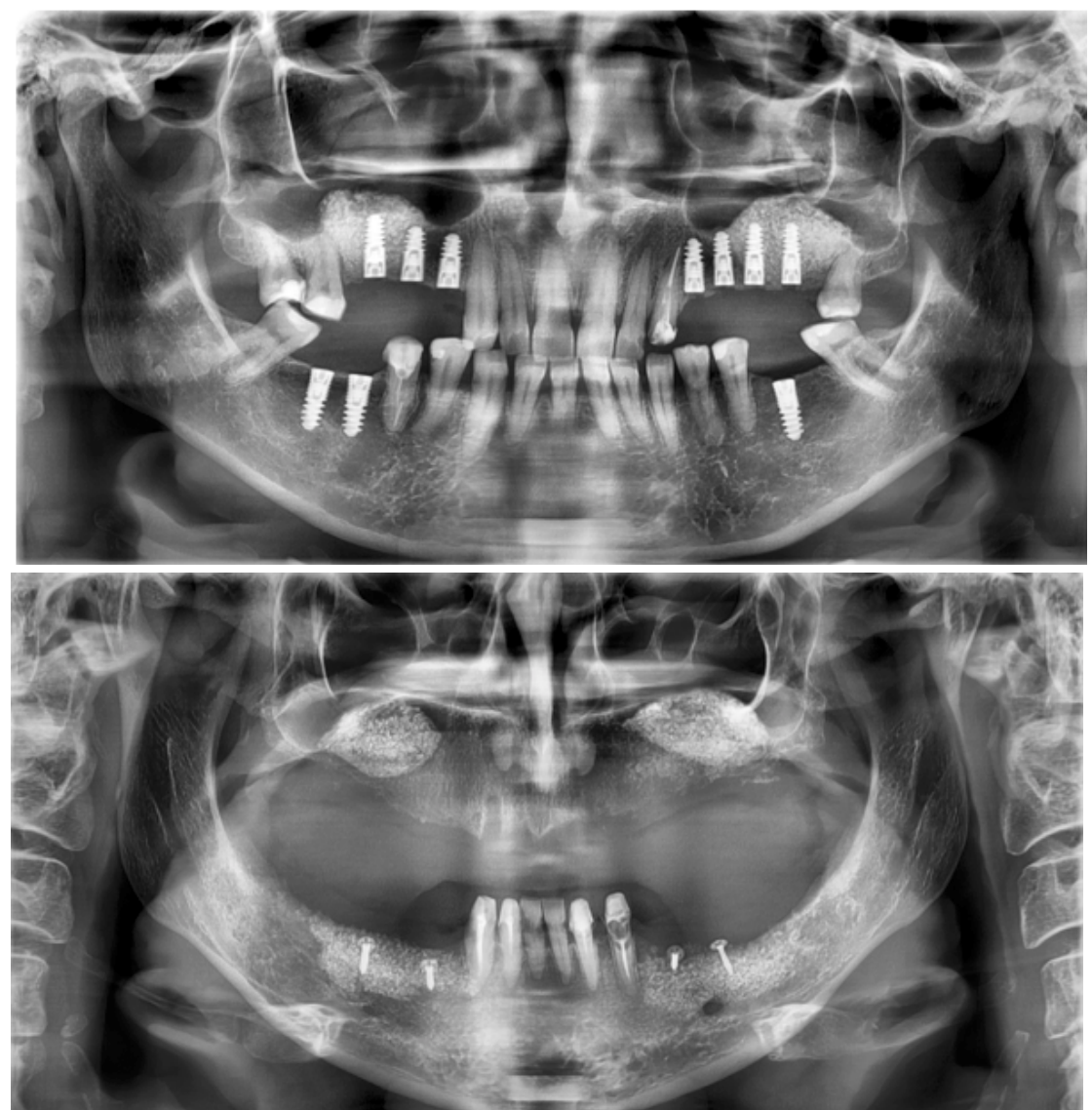

Fig.2. OPG exam clearly reveal bilaterally external maxillary sinuses lifting together with dental status rehabilitation through metallic implants
Fig.3. Postoperative OPG evaluation of a patient with bilaterally external maxillary sinuses lifting together with bilaterally both vertical and horizontal alveolar crest augmentation in order to dental rehabilitation by metallic implants from the world, an innovation in the field, ensuring the minimum invasive sinus lift. In 10 cases, internal maxillary sinuses lifting were done with bone addition (71\%), but in 4 cases were done with only compaction (29\%). Upon patientsex distribution 8 internal maxillary sinus lifting were done in women patients (57\%) and only 6 in men (43\%).

Mandible regional vertical alveolar crest augmentations were done in 9 patients (47\%) and horizontal alveolar crest augmentation in 7 patients (37\%), in 3 patients being necessary both vertical and horizontal alveolar crest augmentation (16\%). Vertical alveolar crestaugmentations were done in quadrant IV in 6 patients (67\%) and in quadrant III in 3 patients (33\%). Horizontal alveolar crest augmentations were done in quadrant IV in 4 patients (57\%) and in quadrant III in 3 patients (43\%). Upon patient sex distribution 6 vertical alveolar crest augmentations were done in men (67\%) and only 3 in women patients (33\%). Horizontal alveolar crest augmentations were done in 4 men patients (57\%) and in 3 women patients (43\%). Internal or external sinus lift and regional bone addition performed for dental status rehabilitation through metallic implants were done by bovine bone addition - Cerabone granulation 1-2 mm which was then covered with a resorbable membrane of collagen - Osgide - resorbable porcine membrane over which the gum suture is performed.

All 47 patients dental rehabilitated by metallic implants were OPG diagnostic assessed with regional alveolar crest atrophy after dental segments extraction, which also guide the implants proper location inserts and finally evaluated the proper alignment, parallelism, direction, spacing and angulations of the metallic dental implants.

\section{Conclusions}

The OPG - exam offer a detailed evaluation of the dentomaxillary region together with the accompanying vital structures: the floor of the sinus, the floor of the nose, the roof of the mandibular canal and the position of the mental neurovascular bundle.

OPG is the imaging modality of choice in the diagnosis algorithms of inflammatory radicular cysts and alveolar crestatrophy offering high abilities in the evaluation of bone tissue loss.

OPG are of great importance value in patients with inflammatory radicular cysts and alveolar crest atrophy being able to offer subtle and no doubt data for precise skeletal loss extent diagnosis and local dental status in order to proceed a clearly quantified treatment with accurate capacities in postoperative evaluation.

The OPG - exam offer a detailed precise evaluation of heterolog membranes and materials integration used in the postoperative osseous defect repair and proper alignment, parallelism, direction, spacing and angulations in patients with dental rehabilitation by metallic implants.

\section{References}

1. REGEZI J.A., SCIUBBA J.; Oral pathology Clinical-pathologic correlations; W. B. Saunders Company USA 1993

2. MARY SCANLON, MD and LAURIE A.LOEVNER, MD Head and neck imaging in: E. SCOTT PRETORIUS, J EFFREY A. SOLOMON, Radiology Secrets, Second Edition, ELSEVIER MOSBY, 2006, pag: 388-393

3. GAHLEITNER A., HOFSCHNEIDER U., PRETTERKLIEBER M., ZAUZA K., WATZEK G.; Lingual vascular canals of the mandible: evaluation with dental CT în Radiology Volume 220, Number 1; July 2001 4. GUY POYTON H., PHAROAH M.J.; Oral radiology; B. C. Decker Inc. Totonto Philadelphia 1989

5. HARNSBERGER H.R.; Handbook of head and neck imaging, second edition; Mosby St. Louis, Missouri, 1995

6. BARAZANCHI A.,LI K., AL-AMLEH B., LYONS K., WADDELL J., Additive Technology: Update on Current Materials and Applications in Dentistry, Journal of Prosthodontics-Implant Esthetic and Reconstructive Dentistry, 2017, 26(2):156-163

Manuscript received: 28.10 .2019 